\title{
Editorial
}

\section{Omicron variant of SARS-CoV-2- A virus of concern}

\author{
Sankalp Yadav $1, *$ \\ ${ }^{1}$ Dept. of Medicine \& Tuberculosis, Shri Madan Lal Khurana Chest Clinic, Moti Nagar, NDMC, New Delhi, India
}

A R T I C L E I N F O

Article history:

Received 30-11-2021

Accepted 16-12-2021

Available online 31-12-2021
This is an Open Access (OA) journal, and articles are distributed under the terms of the Creative Commons Attribution-NonCommercial-ShareAlike 4.0 License, which allows others to remix, tweak, and build upon the work non-commercially, as long as appropriate credit is given and the new creations are licensed under the identical terms.

For reprints contact: reprint@ipinnovative.com
The world is seriously affected by the current pandemic of coronavirus diseases 2019 (COVID-19). ${ }^{1}$ This viral disease has led to extensive morbidity and mortality. ${ }^{1}$ The virus which results in COVID-19 i.e., severe acute respiratory syndrome coronavirus 2 (SARS-CoV-2) is an RNA virus and has a tendency to mutate. ${ }^{2}$ The same is evidenced by the detection of variants of this virus namely alpha, delta, etc. ${ }^{3}$ The latest reports of a new variant of SARS-CoV-2 named by the WHO's Technical Advisory Group on Virus Evolution (TAG-VE) as 'B.1.1.529' or 'Omicron' variant has resulted in panic and instilled fear among the health care authorities and the general public. ${ }^{4}$ This variant was first reported in South Africa on $24^{\text {th }}$ November $2021 .^{5}$ It was detected during a recent steep rise in the cases of COVID19 in South Africa ${ }^{5}$. The first confirmed sample for this variant was collected on $9^{\text {th }}$ November $2021 .^{5}$ Globally till $8^{\text {th }}$ December 2021, there have been 1458 confirmed cases reported by fifty-five countries. ${ }^{6}$

\section{The WHO has two working definitions for SARS-CoV-2} Variant of Interest (VOI) and Variant of Concern (VOC) ${ }^{5}$

'A SARS-CoV-2 VOI is a SARS-CoV-2 variant with genetic changes that are predicted or known to affect virus

\footnotetext{
* Corresponding author.

E-mail address: drsankalpyadav@gmail.com (S. Yadav).
}

characteristics such as transmissibility, disease severity, immune escape, diagnostic or therapeutic escape; and that has been identified as causing significant community transmission or multiple COVID-19 clusters, in multiple countries with increasing relative prevalence alongside an increasing number of cases over time, or other apparent epidemiological impacts to suggest an emerging risk to global public health'. ${ }^{5}$

Again, 'a SARS-CoV-2 VOC is a SARS-CoV-2 variant that meets the definition of a VOI and, through a comparative assessment, has been demonstrated to be associated with one or more of the following changes at a degree of global public health significance: increase in transmissibility or detrimental change in COVID-19 epidemiology; or increase in virulence or change in clinical disease presentation; or decrease in the effectiveness of public health and social measures or available diagnostics, vaccines, therapeutics'. 5

Besides, a Probable case of SARS-CoV-2 Omicron VOC infection is defined as, 'a person with a positive Nucleic Acid Amplification Test (NAAT) or Rapid Antigen Detection Test (RADT) for SARS-CoV-2 and one of the following: S-gene target failure or another PCR-based Single Nucleotide polymorphism (SNP) assay indicative of the SARS-CoV-2 Omicron VOC or is a contact of a probable or confirmed case of SARS-CoV-2 Omicron VOC infection'. 6

The WHO designated this new variant as a VOC post evidence of multiple mutations (more than 30) in the 
original virus genome. ${ }^{7}$ These large-scale mutations could affect the transmissibility and have a higher chance of reinfection in those who had COVID-19 disease. ${ }^{7}$ The same is based on the fact that there is a surge in COVID19 patients in all the provinces of South Africa and this Omicron variant is being detected in the majority of these cases. $^{5}$

The diagnostic techniques available currently for SARSCoV-2 i.e., real-time polymerase chain reaction (RT-PCR) have been useful in detecting this new variant of SARSCoV-2. ${ }^{5}$ There are reports from many labs mentioning that in the PCR test, one of the three target genes goes undetected (called S gene dropout or S gene target failure) and this test can therefore after sequencing confirmation, should be used as a marker for this VOC. ${ }^{5}$ Utilizing this approach of detecting this VOC has been found out at quicker rates than past surges in infection, thereby implying that this VOC may have a growth dominance. 5

To date, a large number of epidemiological studies are underway in areas where this virus is detected and worldwide to determine the exact rate of transmission and severity of this VOC infection (including symptoms), the performance of vaccines, diagnostic tests, and effectiveness of available treatments. ${ }^{4}$

Symptom wise this VOC is considered mild. ${ }^{8}$ The patients present with fatigue, scratchy throat, mild fever which subsides of its own. ${ }^{8}$ Other symptoms include dry cough, night sweats, body pain. ${ }^{8}$ However, there are no reports of high-grade fever, stuffed nose, and loss of taste or smell as previously reported in the infection with other variants of SARS-CoV-2 like alpha and delta. ${ }^{8}$

Currently, it takes at least 36 hours for targeted sequencing and four to five days for whole-genome sequencing to find out this new $\mathrm{VOC}^{9}$. However, there are reports of the development of a hydrolysis probe-based realtime RT-PCR assay from the Indian Council of Medical Research (ICMR) to find out the Omicron variant of the coronavirus in two hours, which could help in the early detection of this $\mathrm{VOC}^{9}$. This is a remarkable achievement as early detection could serve as a boon for prompt treatment ultimately could have a major effect on the survivability of those diagnosed with this VOC.

Also, based on the large-scale rise of cases in South Africa and assuming greater transmissibility of this VOC, the WHO has asked countries to increase surveillance and sequencing efforts to better understand circulating SARSCoV-2 variants. ${ }^{5}$ Provide a complete genome sequencing report and related metadata to a publicly available database, such as GISAID ${ }^{5}$. Promptly record and report initial cases/clusters associated with VOC infection to WHO through the IHR mechanism. ${ }^{5}$ At places with capacity and in unison with the international community, carry out field investigations and laboratory assessments to improve understanding of the potential impacts of the VOC on COVID-19 epidemiology, severity, the effectiveness of public health and social measures, diagnostic methods, immune responses, antibody neutralization, or other important characteristics. ${ }^{5}$

It is also advised that COVID-19 appropriate behavior should be continued to be practiced by all to reduce their risk of infection due to the new VOC, this also includes the practice of manifested public health and social provisions such as the use of well-fitting face masks, maintenance of hand hygiene, use of sanitizers, physical distancing, revamping the ventilation of closed/indoor spaces, avoiding congested spaces, and timely getting the corona vaccines. ${ }^{5}$

To conclude, this new variant of SARS-CoV-2 is a VOC and in the absence of concrete data about this variant, COVID-19 appropriate behavior is the best solution for the general public. The virus is bound to mutate and thus efforts should be aimed at the prevention of the infection. There are several incidences of the lackluster attitude of the general public post vaccination but it should always be kept in mind that vaccine only provides immunity but carelessness could be disastrous. Also, underprivileged countries where total vaccination is very low, like those in Africa with a very high prevalence of HIV should work to increase it for the benefit of its people. International agencies and developed countries that are having surplus vaccines should help these underserved populations.

\section{Acknowlegements}

None.

\section{Conflict of Interests}

There are no conflicts of interests

\section{Source of Funding}

None.

\section{References}

1. Yadav S, Rawal G. Mucormycosis in COVID-19- A burgeoning epidemic in the ongoing pandemic. IP Indian J Immunol Respir Med. 2021;6(2):67-70.

2. Harvey WT, Carabelli AM, Jackson B, Gupta RK, Thomson EC, Harrison EM, et al. SARS-CoV-2 variants, spike mutations and immune escape. Nat Rev Microbiol. 2021;19:409-24. 10010.1038/s41579-(1)2工 $00573-0$.

3. SARS-CoV-2 Variant Classifications and Definitions. Available from URL:- https://www.cdc.gov/coronavirus/2019-ncov/variants/variant-cl assifications.html. Last accessed 2021 on December 12.

4. WHO. Update on Omicron. Available from URL:- https://www.who. int/news/item/28-11-2021-update-on-omicron. Last accessed 2021 on December 12 .

5. WHO. Classification of Omicron (B.1.1.529): SARS-CoV-2 Variant of Concern. Available from URL:- https://www.who.int/news/item/26-11 -2021-classification-of-omicron-(b.1.1.529)-sars-cov-2-variant-of-con cern. Last accessed 2021 on December 12.

6. Epidemiological update: Omicron variant of concern (VOC) - data as of 8 December 2021 (12.00). Available from URL:- https://www.ecdc europa.eu/en/news-events/epidemiological-update-omicron-data-8-dec ember. Last accessed 2021 on December 12. 2021; 
7. PTI. Omicron variant reportedly has over 30 mutations in spike protein, may bypass vaccines: AIIMS chief. Available from URL:- https://w ww.indiatoday.in/coronavirus-outbreak/story/omicron-variant-repor tedly-has-30-mutations-in-spike-protein-may-bypass-vaccines-aiims -chief-randeep-guelria-1881833-2021-11-28. Last accessed 2021 on December 12 .

8. Five symptoms of Omicron variant you should not ignore. Available from URL:- https://www.livemint.com/news/india/five-symptoms-o f-omicron-variant-you-should-not-ignore-11639211738790.html. Last accessed 2021 on December 12 .

9. ICMR team designs kit to detect Omicron variant in 2 hours. Available from URL:- https://www.hindustantimes.com/india-news/icmr-team-d esigns-kit-to-detect-omicron-variant-in-2-hours-101639269025676.ht ml. Last accessed 2021 on December 12.

Cite this article: Yadav S. Omicron variant of SARS-CoV-2- A virus of concern. IP Indian J Immunol Respir Med 2021;6(4):207-209. 